\title{
Quadri interfaces
}

\section{Shifting interface in the spacetime}

\section{Roger Prud'homme ${ }^{1}$}

${ }^{1}$ Sorbonne Université, UPMC Univ Paris 6, UMR CNRS 7190, Institut Jean Le Rond d'Alembert, F-75005, Paris.

RÉSUMÉ. Les équations macroscopiques de bilan des interfaces fluides ont été établies en considérant deux échelles de longueur et moyennant quelques approximations, telles que la conservation du vecteur vitesse mixte et du gradient parallèle à la traversée de la zone interfaciale. Nous généralisons cette méthode au cas des quadri interfaces en présence de champs électromagnétiques. Le passage à l'espace-temps $\mathrm{s}^{\mathrm{i}}$ ii est en effet un moyen d'obtenir une présentation homogène des bilans ${ }^{1}$.

ABSTRACT. The macroscopic balance equations of the fluid interfaces were established by considering two scales of length and by means of some approximations, such as the conservation of the mixed velocity vector and of the gradient parallel to the crossing of the interfacial zone. We generalize this method to the case of four-interfaces in the presence of electromagnetic fields. The transition to space-time is indeed a means of obtaining a homogeneous presentation of the balance sheets.

MOTS-CLÉS. lois de bilan, espace-temps, interfaces, fluides, champs électromagnétiques.

KEYWORDS. balance laws, time-space, interfaces, fluids, electromagnetic fields.

\section{Liste des symboles}

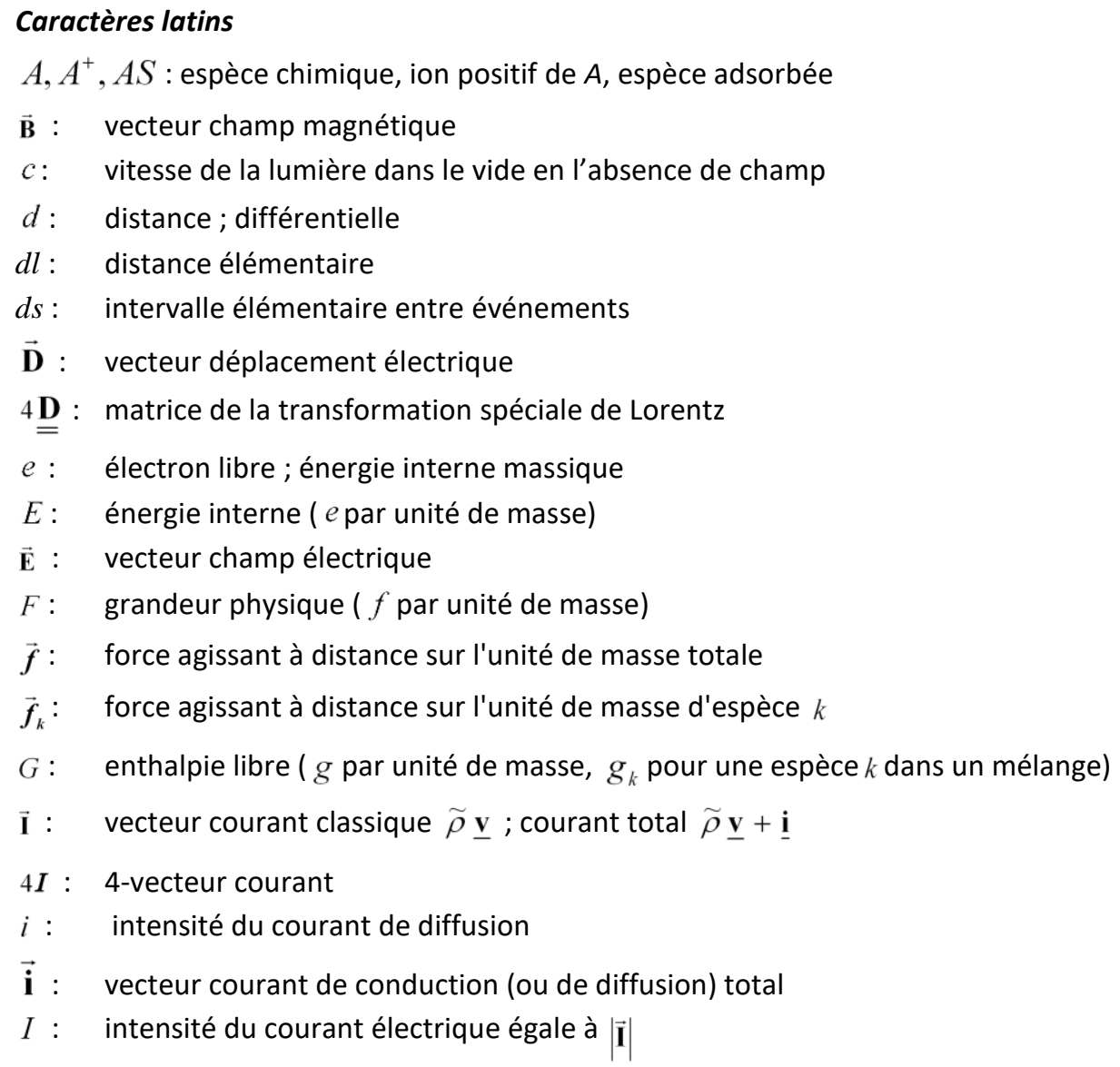

\footnotetext{
${ }^{1}$ Cet article est une mise à jour d'une présentation au Colloque du GDR MFA - Balaruc-Les-Bains - 10/13 novembre 2015.
} 
$\mathbf{J}_{\mathbf{V} F}$ : flux d'une grandeur $F$ par rapport au mouvement de vitesse $\overrightarrow{\mathbf{V}}$

$\mathbf{J}_{F}$ : flux de $F$ par rapport à un référentiel fixe $\overrightarrow{\mathbf{V}}=\overrightarrow{\mathbf{0}}$

$\boldsymbol{J}_{F}$ : flux de $F$ par rapport au mouvement barycentrique du fluide $\overrightarrow{\mathbf{V}}=\overrightarrow{\mathbf{v}}$

$\overrightarrow{\boldsymbol{J}}_{D k}$ : flux de diffusion de l'espèce $k$ égal à $\rho_{k}\left(\mathbf{v}_{k}-\mathbf{v}\right)$

$k: \quad$ espèce chimique; constante de Boltzmann

$K$ : énergie cinétique ( $k$ par unité de masse)

$m: \quad$ masse totale ; masse d'un point matériel $m_{0} / \alpha$

$m_{0}$ : masse d'un point matériel dans l'espace de Minkowski ;

$M$ : masse ; masse moléculaire ; point matériel

$\mathcal{M}$ : masse molaire

M : polarisation magnétique ( $\mathbf{m}=\mathbf{M} / \rho$ : par unité de masse)

$\overrightarrow{4 \mathbf{M}}:$ 4-vecteur correspondant au point matériel

$4 \mathcal{M}:$ 4-tenseur égal à $4 \mathcal{F}-4 \mathcal{H}$

$\dot{m}$ : débit masse par unité d'aire ; débit unitaire

$n: \quad$ nombre total de moles

$n_{k}:$ nombre de moles d'espèce $k$

$N$ : nombre d'espèces ; coordonnée normale d'une interface

$\overrightarrow{\mathbf{n}}, \overrightarrow{\mathbf{N}}, \overrightarrow{4} \mathbf{N}$ : normale unitaire à une surface, 4-normale une surface dans l'espace-temps

$p:$ pression thermodynamique

$\overrightarrow{\mathbf{P}}$ : vecteur polarisation électrique ( $\mathbf{p}=\overrightarrow{\mathbf{P}} / \rho$ : par unité de masse)

$\overrightarrow{\overrightarrow{\mathbf{P}}}$ : tenseur de pression

$4 \mathscr{P}$ : 4-tenseur d'impulsion-énergie

$4 \mathcal{P}^{c h}$ : 4-tenseur $\mathrm{d}^{\prime}$ impulsion-énergie dû au champ électromagnétique (ou 4-tenseur de flux électromagnétique)

$4 \mathcal{P}^{(m)}:$ 4-tenseur d'impulsion-énergie dû à la masse

$\overrightarrow{\mathbf{q}}$ : vecteur flux de chaleur

$R$ : constante molaire universelle des gaz ; rayon ; ou résistance électrique

$\mathfrak{R}: \quad$ référentiel

$S: \quad$ entropie (s par unité de masse)

$S, S:$ surface

$t:$ temps

$T: \quad$ température absolue

$\mathrm{u}, \mathrm{v}, \mathrm{w}$ : composantes de la vitesse $\overrightarrow{\mathbf{v}}$ en coordonnées cartésiennes

$\overrightarrow{\mathbf{v}}$ : vecteur vitesse; vecteur vitesse du point matériel de coordonnées $v_{x}, v_{y}$ et $v_{z}$ de module $v$; vecteur vitesse barycentrique dans un fluide composite $\sum_{k} Y_{k} \overrightarrow{\mathbf{v}}_{k}$ 
$\overrightarrow{\mathbf{v}}_{k}$ : vecteur vitesse de l'espèce $k$

$\overrightarrow{\mathbf{V}}$ : vecteur; vecteur vitesse; vitesse mixte au niveau de l'interface $\overrightarrow{\mathbf{V}}=\overrightarrow{\mathbf{v}}_{/ /}+\overrightarrow{\mathbf{w}}$

$4 \mathbf{V}$ : 4-vitesse du point matériel

$v,(\partial v)$ : volume de contrôle, sa frontière

$\overrightarrow{\mathbf{V}}_{k}: \quad$ vitesse de diffusion des espèces $\overrightarrow{\mathbf{v}}_{k}-\overrightarrow{\mathbf{v}}$

$\overrightarrow{\mathbf{w}}$ : $\quad$ vitesse d'une surface (sa composante normale)

$\dot{W}_{F}, \dot{W}_{F a}$ : taux de production de la grandeur $F$ resp. par unité de volume, par unité d'aire

$\dot{W}_{k}: \quad$ taux de production de l'espèce $k$

$\dot{W}_{E_{t} a}$ : source d'énergie totale par unité d'aire et de temps à l'interface

$x, y, z$ : coordonnées cartésiennes

$X_{k}, Y_{k}$ : fraction molaire, resp. massique, de l'espèce $k$

$z: \quad$ charge massique $\sum_{k} z_{k} Y_{k}$ d'un mélange

$z_{k}: \quad$ charge massique d'espèce $k$

\section{Caractères grecs}

$\alpha:$ quantité $\sqrt{1-v^{2} / c^{2}}$

$\underline{\underline{\varepsilon}}$ : tenseur diélectrique

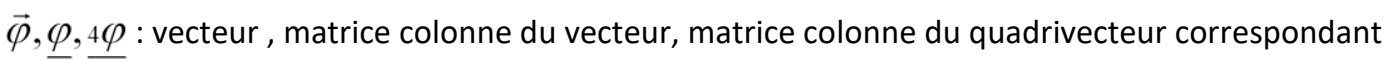

$\overrightarrow{\overrightarrow{\mathbf{T}}}, \underline{\underline{\mathbf{T}}}, \underline{\underline{4}}$ : tenseur d'ordre 2, matrice de ce tenseur, matrice d'un quadri tenseur

${ }_{4} \vec{\Phi}: \quad$ 4-vecteur force

$\overrightarrow{\vec{\Pi}}$ : tenseur de pressions visqueuses

$\rho$ : masse volumique

$\rho_{j}:$ masse volumique partielle de l'espèce $j$

$\tau: \quad$ temps propre d'un point matériel dans l'espace de Minkowski égal à $\alpha t$

$\tilde{\rho}: \quad$ charge classique par unité de volume $\widetilde{\rho}_{0} / \alpha$

$\tilde{\rho}_{0}$ : charge électrique dans l'espace de Minkowski

$\widetilde{\rho}_{k}$ : charge de l'espèce $k$ par unité de volume

$\vartheta: \quad$ volume par unité de masse égal à $1 / \rho$

\section{Autres symboles, indices, exposants}

${ }_{a}: \quad$ indice désignant une quantité extensive par unité d'aire

${ }^{c h}$ : exposant désignant une quantité de type champ

${ }^{m}: \quad$ exposant désignant une quantité de type masse

$s: \quad$ indice désignant une quantité intensive ou spécifique au niveau de l'interface

${ }^{T}$ : $\quad$ exposant indiquant la transposition d'un tenseur, d'une matrice

$\vec{\nabla}: \quad$ gradient (nabla)

$/ /, \perp$ : parallèlement, resp. perpendiculairement à une surface

$\overrightarrow{\overrightarrow{1}}$ : tenseur unité 


\section{Introduction}

Résumons les objectifs de cet article. Les problématiques traitées sont les suivantes :

1. Écrire correctement les équations aux interfaces dans l'espace 4D de Minkowski.

2. Écrire ces équations en présence d'un champ électromagnétique.

L'intérêt d'une formulation $4 D$ est de : Obtenir des formulations tensorielles; tenir compte, si besoin est, de la relativité.

La méthode adoptée consiste en trois points :

- On rappelle la méthode utilisée dans le cas 3D pour les fluides en l'absence de champ, d'abord dans le volume, puis à l'interface

- Les règles de la relativité restreinte sont ensuite rappelées pour être appliquées aux bilans dans le quadri-volume, puis à l'interface

- Les tenseurs électromagnétiques sont ensuite définis, ce qui permet d'établir les bilans dans les milieux fluides en présence de champs

\section{Rappel concernant les interfaces en coordonnées 3D}

\subsection{Bilans en volume}

Il est intéressant d'écrire les équations de bilan dans le volume en suivant le mouvement ayant un champ de vitesse connu. $F$ étant une propriété physique extensive (masse, quantité de mouvement, énergie, etc.) d'un fluide (plus généralement, d'un milieu continu), de valeur $f$ par unité de masse, on peut écrire la forme intégrale du bilan de $F$ dans le volume :

$$
\frac{d}{d t} \int_{\mathcal{V}} \rho f d \mathcal{V}+\int_{\partial \mathcal{V}} \vec{\jmath}_{F} \cdot \vec{n} d S=\int_{\mathcal{V}} \dot{W}_{F} d \mathcal{V}
$$

$(\mathcal{V})$ : volume de contrôle de surface limite $(\partial \mathcal{V})$ ayant $\vec{n}$ pour normale extérieure (Figure 1).

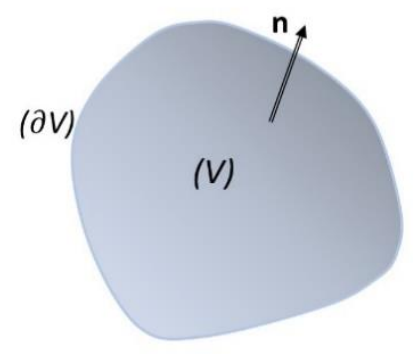

Figure 1. Volume de contrôle connexe et normale unitaire à sa frontière.

Pour toute grandeur $F$ ( $f$ par unité de masse), nous écrirons donc localement :

$\frac{d_{\mathbf{V}}(\rho f)}{d t}+\rho f \vec{\nabla} \cdot \overrightarrow{\mathbf{V}}+\vec{\nabla} \cdot \overrightarrow{\mathbf{J}}_{\mathbf{V} F}=\dot{W}_{F}$ 
avec les définitions suivantes de la dérivée en suivant le mouvement de vitesse $\overrightarrow{\mathbf{V}}$, et du vecteur flux : $\frac{d_{\mathbf{V}}}{d t}=\frac{\partial}{\partial t}+\overrightarrow{\mathbf{V}} \cdot \vec{\nabla}, \overrightarrow{\mathbf{J}}_{\mathbf{V} F}=\vec{\jmath}_{F}+\rho f(\overrightarrow{\mathbf{v}}-\overrightarrow{\mathbf{V}})$, le flux $\overrightarrow{\boldsymbol{J}}_{F}$ étant celui du mouvement barycentrique (vitesse $\overrightarrow{\mathbf{v}}$ ). Le taux de production de l'unité de volume de la grandeur $F$ est noté $\dot{W}_{F}$.

On déduit directement de [1] par exemple le bilan de la masse d'un fluide simple : $\frac{d_{\mathbf{V}} \rho}{d t}+\rho \vec{\nabla} \cdot \overrightarrow{\mathbf{V}}+\vec{\nabla} \cdot \rho(\overrightarrow{\mathbf{v}}-\overrightarrow{\mathbf{V}})=0 . \quad$ En $\quad$ prenant $\overrightarrow{\mathbf{V}}=\overrightarrow{\mathbf{v}}: \quad \frac{d \rho}{d t}+\rho \vec{\nabla} \cdot \overrightarrow{\mathbf{v}}=0$, et en prenant $\quad \overrightarrow{\mathbf{V}}=\overrightarrow{\mathbf{0}}:$ $\frac{\partial \rho}{\partial t}+\vec{\nabla} \cdot(\rho \overrightarrow{\mathbf{v}})=0$. Ces relations restent valables pour un mélange de masse volumique totale $\rho$ et de vitesse barycentrique $\overrightarrow{\mathbf{v}}$.

Les bilans de quantité de mouvement et d'énergie vérifieront aussi des équations de la forme [1], de même que le bilan de l'entropie. Il s'agit là de "grandeurs de type masse".

Ajoutons le bilan de charge électrique pour les milieux conducteurs : $\rho d z / d t+\vec{\nabla} \cdot \overrightarrow{\mathbf{i}}=0$

Le cas des grandeurs caractérisant le champ électromagnétique traitées en section 2.3 et 2.4 , nécessite un traitement spécifique. Les équations de bilan de ces "grandeurs de type champ" dépendront en particulier de la polarisation du milieu ${ }^{\text {iii }}$.

\subsection{Bilans aux interfaces}

Pour les zones interfaciales, a priori mobiles et déformables, il faut définir un champ de vitesse mixte $\overrightarrow{\mathbf{V}}$ particulier.

La vitesse mixte considérée à la traversée de la zone interfaciale, schématisée sur la Figure 2, limitée par les surfaces $S^{-}$et $S^{+}$, est $: \overrightarrow{\mathbf{v}}=\overrightarrow{\mathbf{v}}_{/ /}+\overrightarrow{\mathbf{w}}$, et nous ferons des hypothèses spécifiques de conservation à la traversée entre les coordonnées normales $N$ et $N^{+}$.

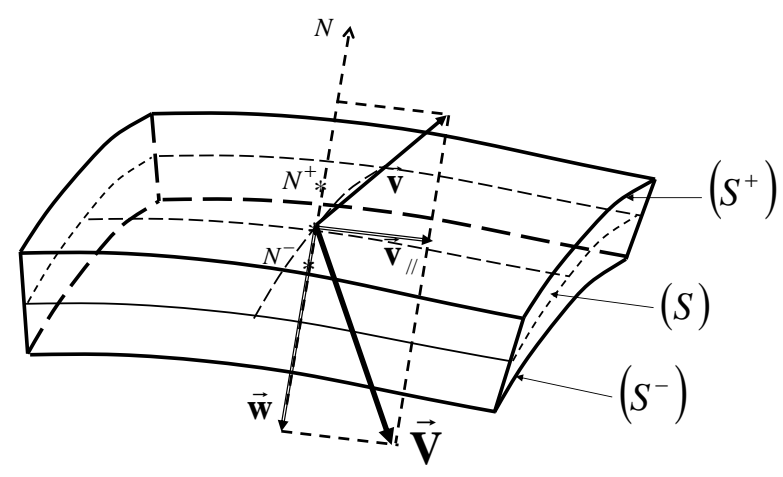

Figure 2. Représentation d'une zone interfaciale à petite échelle au voisinage d'un point courant (*) de la surface moyenne (S), de normale $\overrightarrow{\mathbf{N}}$ orientée de (-) vers (+). On définit la vitesse mixte $\overrightarrow{\mathbf{v}}=\overrightarrow{\mathbf{v}} / /+\overrightarrow{\mathbf{w}}$, où $\overrightarrow{\mathbf{v}}_{/ /}$ est la composante du vecteur vitesse barycentrique $\overrightarrow{\mathbf{v}} d u$ fluide parallèle à l'interface, et $\overrightarrow{\mathbf{w}}$ la vitesse normale de déplacement de l'interface. $N$ désigne l'ordonnée suivant la normale $\overrightarrow{\mathbf{N}}$. Les ordonnées au point courant de $(S)$ et des surfaces limites $\left(S^{-}\right)$et $\left(S^{+}\right)$, sont respectivement $N=N^{*}=0, N=N^{-}$et $N=N^{+}$. 
Dans cette définition interviennent deux mouvements de natures différentes : d'une part la composante parallèle à l'interface du vecteur vitesse barycentrique du fluide $\overrightarrow{\mathbf{v}}_{/ /}$et d'autre part la vitesse normale de déplacement $\overrightarrow{\mathbf{w}}$ de l'interface ${ }^{\mathrm{iv}}$, ce qui explique la dénomination de vitesse mixte.

Le bilan local d'interface s'obtient par intégration entre $N$ et $N^{+}$de l'équation [1] écrite sous la forme $: \frac{d_{\mathbf{V}}(\rho f)}{d t}+\rho f \vec{\nabla}_{/ /} \cdot \overrightarrow{\mathbf{V}}+\vec{\nabla}_{/ /} \cdot \overrightarrow{\mathbf{J}}_{\mathbf{V} F}+\frac{\partial \overrightarrow{\mathbf{J}}_{\mathbf{V} F}}{\partial N}=\dot{W}_{F}$, en supposant la conservation du vecteur vitesse mixte $\overrightarrow{\mathbf{V}}$ et la conservation du gradient parallèle $\vec{\nabla}_{/ /}$. Les expressions de $d_{\mathbf{V}} / d t$ et de $\overrightarrow{\mathbf{J}}_{\mathbf{V} F}$ dépendent de ce champ de vitesse $\overrightarrow{\mathbf{V}}(x, y, z, t)$.

En posant :

$$
\rho_{a}=\int_{N^{-}}^{N^{+}} \rho d N, \rho_{a} f_{S}=\int_{N^{-}}^{N^{+}} \rho f d N, \quad \overrightarrow{\mathbf{J}}_{\mathbf{V} F a}=\int_{N^{+}}^{N^{+}} \overrightarrow{\mathbf{J}}_{\mathbf{V} F} d N, \dot{W}_{F a}=\int_{N^{-}}^{N^{+}} \dot{W}_{F} d N
$$

on obtient :

$$
\frac{d_{S}\left(\rho_{a} f_{S}\right)}{d t}+\rho_{a} f_{S} \vec{\nabla}_{S} \cdot \overrightarrow{\mathbf{v}}_{S}+\vec{\nabla}_{S} \cdot \overrightarrow{\mathbf{J}}_{\mathbf{V} F a}+\left[J_{\mathbf{V} F \perp}\right]_{-}^{+}=\dot{W}_{F a}
$$

avec $: \overrightarrow{\mathbf{V}}=\overrightarrow{\mathbf{V}}_{S}, \vec{\nabla}_{/ /}=\vec{\nabla}_{S}, d_{S} / d t=\partial / \partial t+\overrightarrow{\mathbf{V}}_{S} \cdot \vec{\nabla}, \varphi_{\perp}=\vec{\varphi} \cdot \overrightarrow{\mathbf{N}},[\varphi]_{-}^{+}=\varphi_{+}-\varphi_{-}, \quad \overrightarrow{\mathbf{N}}$ étant la normale unitaire à l'interface.

On doit aussi écrire des conditions de cohérence Indiquant que les composantes normales des tenseurs d'interface sont nulles. Ainsi : $\overrightarrow{\mathrm{F}}_{\mathrm{F} a} \cdot \overrightarrow{\mathbf{N}}=0$.

\section{Les équations de bilan en volume dans l'espace-temps de Minkowski}

\subsection{Généralités ${ }^{v}$ (relativité restreinte ${ }^{2}$, espace-temps ${ }^{3}$ )}

En matière de relativité, nous nous limiterons à la relativité restreinte et aux référentiels galiléens de vitesses $V$ faibles, telles que $V / c<<1$. L'utilisation des coordonnées d'espace-temps et de la transformation de Lorentz sont de rigueur.

La transformation de Lorentz conserve les intervalles (alors qu'en mécanique classique, les changements de référentiels d'inertie conservent les distances). Cette transformation, présentée dans les annexes A1 et A2, est en fait une rotation dans l'espace-temps.

Lorsque l'on introduit une grandeur physique tensorielle, il faut s'assurer que son expression obéit à la transformation de Lorentz lors des changements de référentiels d'inertie.

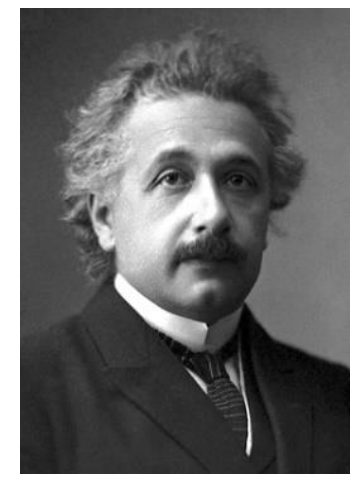

Albert Einstein (1879-1955)

Dans un premier temps nous exprimerons les bilans 3D de la section 1 dans l'espace-temps. Rappelons que les résultats de cette section ont été établis en l'absence de champ électromagnétique;

\footnotetext{
${ }^{2}$ Principe de relativité d'Einstein : la vitesse de propagation des interactions est constante et égale à la vitesse de la lumière dans le vide $c$. En mécanique relativiste, ce sont les intervalles qui se conservent lors des changements de référentiels d'inertie.

${ }^{3}$ Dans l'espace-temps, l'intervalle élémentaire entre événements est défini par : $d s^{2}=c^{2} d t^{2}-d l^{2}$, où $d l$ est la distance élémentaire telle que : $d l^{2}=d x^{2}+d y^{2}+d z^{2}$.
} 
rappelons qu'il s'agit de grandeurs matérielles de type masse. Les équations de bilan 4D obtenues seront donc équivalentes aux équations 3D classiques.

Dans un second temps nous nous occuperons des milieux en présence de champ électromagnétique. Nous introduirons alors des quadri tenseurs électromagnétiques et nous aurons à exprimer les équations de Maxwell dans l'espace de Minkowski.

Nous serons alors en mesure d'exprimer bilans 4D en présence de champ électromagnétique.

\subsection{Bilans 4D dans le volume pour les grandeurs du type masse}

Le quadrivecteur vitesse $4 \overrightarrow{\mathbf{V}}$ est définie par la matrice colonne ${ }_{\underline{4}} \mathbf{V}=\left[\begin{array}{c}\overrightarrow{\mathbf{V}} / \alpha \\ i c / \alpha\end{array}\right], \alpha=\sqrt{1-\frac{|\mathbf{V}|^{2}}{c^{2}}}$. De même, on définit le quadri gradient ${ }^{4}$ comme suit $: \underline{4 \nabla}==\left[\frac{\nabla}{(1 / i c)(\partial / \partial t)}\right]$.

Le bilan local de quadri volume s'écrit alors :

$4 \vec{\nabla}_{\mathbf{V}} \cdot 4 \overrightarrow{\mathbf{J}}_{\mathbf{V} F}+\rho f 4 \vec{\nabla}_{\mathbf{V}} \cdot 4 \overrightarrow{\mathbf{V}}=4 \dot{W}_{F}$

Et le bilan local de quadri interface devient :

$$
4 \vec{\nabla}_{S} \cdot 4 \overrightarrow{\mathbf{J}}_{V F a}+\rho_{a} f_{S} 4 \vec{\nabla}_{S} \cdot 4 \overrightarrow{\mathbf{V}}_{S}+\left[4 \mathbf{J}_{V F \perp}\right]_{-}^{+}=4 \dot{W}_{F a}
$$

avec $: \underline{4 \mathbf{J}_{\mathbf{V} F}}=\left[\begin{array}{l}\underline{\mathbf{J}}_{\mathbf{V} F} / c \\ i \rho f\end{array}\right], \underline{4 \mathbf{J}_{\mathbf{V} F a}}=\left[\begin{array}{l}\underline{\mathbf{J}}_{\mathbf{V} F a} / c \\ i \rho_{a} f_{S}\end{array}\right], 4 \nabla_{S}=\left[\frac{\nabla_{S}}{(1 / i c) d_{S} / d t}\right], 4 \mathbf{J}_{\mathbf{V} F \perp}=4 \overrightarrow{\mathbf{J}}_{\mathbf{V} F} \cdot 4 \overrightarrow{\mathbf{N}}, \underline{4 \mathbf{N}}=\left[\begin{array}{l}\underline{\mathbf{N}} \\ i \overrightarrow{\mathbf{V}}_{S} \cdot \overrightarrow{\mathbf{N}} / c\end{array}\right]$ $4 \dot{W}_{F}=\dot{W}_{F} / c$, respectivement pour les quadri flux, quadri gradients et quadri productions.

Il faut ajouter des conditions de cohérence telles que $: 4 \vec{\jmath}_{\mathbf{F} a} \cdot 4 \overrightarrow{\mathbf{N}}=0$.

\subsection{Quadri tenseurs électromagnétiques dans le volume}

- Champs et déplacements électromagnétiques

On introduit d'abord les trois quadri tenseurs suivants, contenant les composantes des vecteurs champs et déplacements électromagnétiques : $\overrightarrow{\mathbf{E}}, \overrightarrow{\mathbf{D}}$ pour la partie champ électrique et $\overrightarrow{\mathbf{B}}, \overrightarrow{\mathbf{H}}$ pour la partie magnétique. Les matrices correspondant à ces quadri tenseurs s'écrivent dans le référentiel donné :

$$
\underline{\underline{4 \boldsymbol{F}}}=\left[\begin{array}{cccc}
0 & B_{z} & -B_{y} & -i E_{x} \\
-B_{z} & 0 & B_{x} & -i E_{y} \\
B_{y} & -B_{x} & 0 & -i E_{z} \\
i E_{x} & i E_{y} & i E_{z} & 0
\end{array}\right], \underline{\underline{4 F^{\star}}}=\left[\begin{array}{cccc}
0 & -i E_{z} & i E_{y} & B_{x} \\
i E_{z} & 0 & -i E_{x} & B_{y} \\
-i E_{y} & i E_{x} & 0 & B_{z} \\
-B_{x} & -B_{y} & -B_{z} & 0
\end{array}\right]
$$

\footnotetext{
${ }^{4}$ Le laplacien $\Delta=\nabla^{2}=\frac{\partial^{2}}{\partial x^{2}}+\frac{\partial^{2}}{\partial y^{2}}+\frac{\partial^{2}}{\partial z^{2}}$ devient en 4 D le d'Alembertien $\square={ }_{4} \nabla^{2}=\frac{\partial^{2}}{\partial x^{2}}+\frac{\partial^{2}}{\partial y^{2}}+\frac{\partial^{2}}{\partial z^{2}}-\frac{1}{c^{2}} \frac{\partial^{2}}{\partial t^{2}}$
} 
$\underline{\underline{4 \mathcal{H}}}=\left[\begin{array}{cccc}0 & H_{z} & -H_{y} & -i D_{x} \\ -H_{z} & 0 & H_{x} & -i D_{y} \\ H_{y} & -H_{x} & 0 & -i D_{z} \\ i D_{x} & i D_{y} & i D_{z} & 0\end{array}\right]$

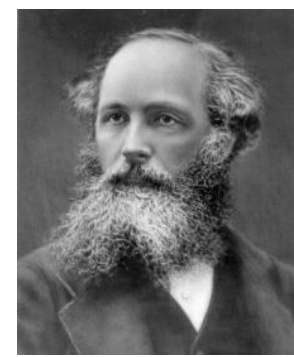

James Clerk Maxwell (1831-1879)

Les tenseurs électromagnétiques $4 \overrightarrow{\overrightarrow{\mathcal{H}}}$ et $4 \overrightarrow{\overrightarrow{\mathcal{F}}}^{\star}$ vérifient les expressions $4 D$ des équations de Maxwell :

$4 \vec{\nabla} \cdot 4 \overrightarrow{\mathcal{H}}=4 \vec{I}, 4 \vec{\nabla} \cdot 4 \overrightarrow{\vec{F}^{\star}}=\overrightarrow{0}$

On considère de plus le quadri tenseur d'impulsion-énergie : $4 \overrightarrow{\overrightarrow{\mathcal{P}}}=4 \overrightarrow{\overrightarrow{\mathcal{P}}}^{m}+4 \overrightarrow{\overrightarrow{\mathcal{P}}}^{c h}$, avec sa partie due $\grave{a}$ la masse et sa partie due aux champs. Ces quadri tenseurs représentent des flux de quantité de mouvement-énergie. $4 \overrightarrow{\overrightarrow{\mathcal{P}}}^{c h}$ est fonction des champs déplacements électriques et magnétiques : $\overrightarrow{\mathbf{E}}, \overrightarrow{\mathbf{B}}, \overrightarrow{\mathbf{D}}, \overrightarrow{\mathbf{H}}$ et du vecteur vitesse $\overrightarrow{\mathbf{v}}$ rapporté à la vitesse $c$ de la lumière dans le vide.

On définit les tenseurs et matrices correspondantes qui suivent :

- Quadri tenseur d'impulsion énergie dû̀ à la masse, dans le volume :

$\stackrel{4 \mathcal{P}^{m}}{=}=\left[\begin{array}{ll}\rho \overrightarrow{\mathbf{v}} \otimes \overrightarrow{\mathbf{v}}+\overrightarrow{\overrightarrow{\mathbf{P}}} & i c \rho \overrightarrow{\mathbf{v}} \\ \frac{i}{c}[\overrightarrow{\mathbf{q}}+\overrightarrow{\overrightarrow{\mathbf{P}}} \cdot \overrightarrow{\mathbf{v}}+\rho(e+k) \overrightarrow{\mathbf{v}}] & -\rho(e+k)\end{array}\right]$

- Quadri tenseur de pression énergie dû à la masse, à l'interface :

$\stackrel{4 \mathscr{P}}{=}_{a}^{m}=\left[\begin{array}{lr}\rho_{a} \overrightarrow{\mathbf{v}}_{S} \otimes \overrightarrow{\mathbf{v}}_{S}+\overrightarrow{\overrightarrow{\mathbf{P}}}_{a} & \text { ic } \rho_{a} \overrightarrow{\mathbf{v}}_{S} \\ \frac{i}{c}\left[\overrightarrow{\mathbf{q}}_{a}+\overrightarrow{\overrightarrow{\mathbf{P}}}_{a} \cdot \overrightarrow{\mathbf{v}}_{S}+\rho_{a}\left(e_{S}+k_{S}\right) \overrightarrow{\mathbf{v}}_{S}\right] & -\rho_{a}\left(e_{S}+k_{S}\right)\end{array}\right]$

- Quadri tenseur d'impulsion-énergie dî au champ

$$
\underline{\underline{4 \boldsymbol{P}^{c h}}}=\left[\begin{array}{lc}
\underline{\underline{\mathbf{P}^{c h}}} & i \underline{\mathbf{E} \times \underline{\mathbf{H}}} \\
(i / c) \underline{\mathbf{J}_{E}^{c h}} & -E^{c h}
\end{array}\right]
$$

Pour le fluide en mouvement à la vitesse barycentrique $\overrightarrow{\mathbf{v}}$ :

$$
\begin{aligned}
& \overrightarrow{\overrightarrow{\mathbf{P}}}^{c h}=\left(\frac{|\overrightarrow{\mathbf{E}}|^{2}+|\overrightarrow{\mathbf{B}}|^{2}}{2}-\overrightarrow{\mathbf{M}} \cdot \overrightarrow{\mathbf{B}}\right) \overrightarrow{\mathbf{i}}-\overrightarrow{\mathbf{D}} \otimes \overrightarrow{\mathbf{E}}-\overrightarrow{\mathbf{B}} \otimes \overrightarrow{\mathbf{H}}-\frac{\overrightarrow{\mathbf{v}}}{c} \otimes(\overrightarrow{\mathbf{P}} \times \overrightarrow{\mathbf{B}}-\overrightarrow{\mathbf{M}} \times \overrightarrow{\mathbf{E}}) \\
& \overrightarrow{\mathbf{J}}_{E}^{c h}=c(\overrightarrow{\mathbf{E}} \times \overrightarrow{\mathbf{H}})-(\overrightarrow{\mathbf{P}} \cdot \overrightarrow{\mathbf{E}}+\overrightarrow{\mathbf{M}} \cdot \overrightarrow{\mathbf{B}}) \overrightarrow{\mathbf{v}},
\end{aligned}
$$

Le produit vectoriel $\overrightarrow{\mathbf{E}} \times \overrightarrow{\mathbf{H}}$ est dit "vecteur de Poynting". 


$$
E^{c h}=\frac{|\overrightarrow{\mathbf{E}}|^{2}+|\overrightarrow{\mathbf{B}}|^{2}}{2}-\overrightarrow{\mathbf{M}} \times \overrightarrow{\mathbf{B}}-\frac{\overrightarrow{\mathbf{v}}}{c} \cdot(\overrightarrow{\mathbf{P}} \times \overrightarrow{\mathbf{B}}-\overrightarrow{\mathbf{M}} \times \overrightarrow{\mathbf{E}})
$$

les champs et déplacements étant ici mesurés dans le référentiel au repos (d'après de Groot \& Mazur) et dans le cas non relativiste.

- Quadri vecteur des forces extérieures:

$$
\begin{aligned}
\underline{4 \Phi} & =\left[\begin{array}{l}
\rho_{k} \vec{f}_{k} \\
\frac{i}{c} \rho_{k} \vec{f}_{k} \cdot \overrightarrow{\mathbf{v}}_{k}
\end{array}\right] \\
4 \overrightarrow{\boldsymbol{\Phi}} & =\sum_{k} 4 \overrightarrow{\boldsymbol{\Phi}}_{k}
\end{aligned}
$$

\subsection{Bilan 4D d'impulsion-énergie dans le volume}

On écrit que le flux sortant d'impulsion-énergie à travers la frontière du quadri volume considéré est égal au quadri vecteur des forces extérieures, ce qui donne localement :

$$
4 \vec{\nabla} \cdot\left(\overrightarrow{\overrightarrow{\mathcal{P}}}^{c h}+{ }_{4} \overrightarrow{\overrightarrow{\mathcal{P}}}^{m}\right)={ }_{4} \vec{\Phi}
$$

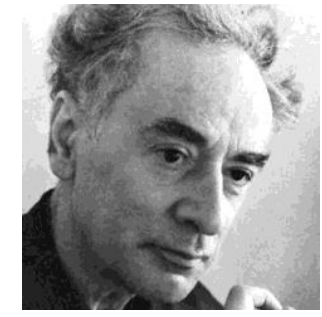

Lev Davidovitch

Landau (1908-1968

\section{Les équations de bilan à l'interface dans l'espace-temps de Minkowski}

Utilisons pour les interfaces avec champ électromagnétique, la même organisation que pour le volume en section 2 : connaissant les bilans d'interface en l'absence de champ électromagnétique, on définit les grandeurs électromagnétiques d'interface et les tenseurs d'impulsion-énergie. Puis on s'intéresse ensuite aux équations de Maxwell et aux bilans généraux en se limitant au cas des fluides simples.

\subsection{Quadri tenseurs électromagnétiques}

Nous donnons dans ce qui suit les définitions et équations de base.

\section{- Champs et déplacements électromagnétiques}

Comme dans le cas du volume, on peut définir des grandeurs d'interface, qui devront vérifier les équations de Maxwell et des conditions de cohérence.

$$
\stackrel{4 \mathcal{F}}{=} a=\left[\begin{array}{cccc}
0 & B_{a z} & -B_{a y} & -i E_{a x} \\
-B_{a z} & 0 & B_{a x} & -i E_{a y} \\
B_{a y} & -B_{a x} & 0 & -i E_{a z} \\
i E_{a x} & i E_{a y} & i E_{a z} & 0
\end{array}\right], \quad \stackrel{4 \mathcal{F}_{a}}{=}{ }^{\star}=\left[\begin{array}{cccc}
0 & -i E_{a z} & i E_{a y} & B_{a x} \\
i E_{a z} & 0 & -i E_{a x} & B_{a y} \\
-i E_{S y} & i E_{a x} & 0 & B_{a z} \\
-B_{a x} & -B_{a y} & -B_{a z} & 0
\end{array}\right]
$$




$$
\stackrel{4 \mathcal{H}}{a}_{a}=\left[\begin{array}{cccc}
0 & H_{a z} & -H_{a y} & -i D_{a x} \\
-H_{a z} & 0 & H_{a x} & -i D_{a y} \\
H_{a y} & -H_{a x} & 0 & -i D_{a z} \\
i D_{a x} & i D_{a y} & i D_{a z} & 0
\end{array}\right]
$$

Les grandeurs d'interface sont en milieu polarisé : $\mathbf{H}_{a}$ vecteur déplacement magnétique $; \mathbf{D}_{a}$ : vecteur déplacement électrique ; $\mathbf{B}_{a}$ : champ magnétique; $\mathbf{E}_{a}$ : champ électrique $; \overrightarrow{\mathbf{P}}_{a}=\mathbf{D}_{a}-\mathbf{E}_{a}$ : polarisation électrique ( $\mathbf{p}_{S}$ par unité de masse); $\mathbf{M}_{a}=\mathbf{B}_{a}-\mathbf{H}_{a}$ : polarisation magnétique ( $\mathbf{m}_{S}$ par unité de masse).

- Équations de Maxwell à l'interface et conditions de cohérence :

$$
4 \vec{\nabla} \cdot 4 \overrightarrow{\mathcal{H}}_{a}=4 \vec{I}_{a}, 4 \vec{\nabla} \cdot 4 \overrightarrow{\vec{F}}_{a}^{\star}=\overrightarrow{\mathbf{0}}
$$

Conditions de cohérence :

$$
4 \overrightarrow{\mathcal{H}}_{a} \cdot 4 \overrightarrow{\mathbf{N}}=4 \overrightarrow{\overrightarrow{\mathcal{F}}}_{a}{ }_{a} \cdot 4 \overrightarrow{\mathbf{N}}=\overrightarrow{\mathbf{0}}, \quad{ }_{4} \overrightarrow{\mathbf{I}}_{a} \cdot 4 \overrightarrow{\mathbf{N}}=0
$$

- Quadri-tenseurs d'impulsion-énergie à l'interface et conditions de cohérence :

$$
\begin{aligned}
& \stackrel{\mathcal{P}}{=}_{a}^{m}=\left[\begin{array}{lr}
\rho_{a} \overrightarrow{\mathbf{v}}_{S} \otimes \overrightarrow{\mathbf{v}}_{S}+\overrightarrow{\overrightarrow{\mathbf{P}}}_{a} & i c \rho_{a} \overrightarrow{\mathbf{v}}_{S} \\
\frac{i}{c}\left[\overrightarrow{\mathbf{q}}_{a}+\overrightarrow{\overrightarrow{\mathbf{P}}}_{a} \cdot \overrightarrow{\mathbf{v}}_{S}+\rho_{a}\left(e_{S}+k_{S}\right) \overrightarrow{\mathbf{v}}_{S}\right] & -\rho_{a}\left(e_{S}+k_{S}\right)
\end{array}\right] \\
& \stackrel{4 \mathcal{P}}{=}_{a}^{c h}=\left[\begin{array}{lr}
\overrightarrow{\overrightarrow{\mathbf{P}}}_{a}^{c h} & i c\left(\rho_{a} \overrightarrow{\mathbf{v}}_{S}+\frac{1}{c} \overrightarrow{\mathbf{E}}_{a} \times \overrightarrow{\mathbf{H}}_{a}\right) \\
(i / c) \overrightarrow{\mathbf{J}}_{E a}^{c h} & -E_{a}^{c h}
\end{array}\right]
\end{aligned}
$$

Conditions de cohérence :

$$
\begin{aligned}
& { }_{4} \overrightarrow{\overrightarrow{\mathcal{P}}}_{a}^{m} \cdot{ }_{4} \overrightarrow{\mathbf{N}}=\overrightarrow{\mathbf{0}}, \quad 4 \overrightarrow{\overrightarrow{\mathcal{P}}}_{a}^{c h} \cdot{ }_{4} \overrightarrow{\mathbf{N}}=\mathbf{\mathbf { 0 }},{ }_{4} \overrightarrow{\boldsymbol{J}}_{F a} \cdot 4 \overrightarrow{\mathbf{N}}=0, \quad 4 \overrightarrow{\mathbf{J}}_{F a} \cdot 4 \overrightarrow{\mathbf{N}}=0, \\
& { }_{4} \overrightarrow{\mathcal{H}}_{a} \cdot 4 \overrightarrow{\mathbf{N}}=\overrightarrow{\mathbf{0}}, \quad{ }_{4} \overrightarrow{\overrightarrow{\mathcal{F}}}_{a}^{\star} \cdot \overrightarrow{\mathbf{N}}=\overrightarrow{\mathbf{0}} \text { et }{ }_{4} \overrightarrow{\overrightarrow{\mathcal{P}}}^{m} \cdot 4 \overrightarrow{\mathbf{N}}=\overrightarrow{\mathbf{0}}, \quad{ }_{4} \overrightarrow{\overrightarrow{\mathcal{P}}}^{c h} \cdot{ }_{4} \overrightarrow{\mathbf{N}}=\overrightarrow{\mathbf{0}}
\end{aligned}
$$

Soit, en développant dans l'espace à trois dimensions :

$$
\begin{aligned}
& \left\{\begin{array}{l}
\left(\rho_{a} \overrightarrow{\mathbf{v}}_{S} \otimes \overrightarrow{\mathbf{v}}_{S}+\overrightarrow{\overrightarrow{\mathbf{P}}}_{a}\right) \cdot \overrightarrow{\mathbf{N}}-V_{S \perp} \rho_{a} \overrightarrow{\mathbf{v}}_{S}=\overrightarrow{\mathbf{0}} \\
{\left[\overrightarrow{\mathbf{q}}_{a}+\overrightarrow{\mathbf{v}}_{S} \cdot \overrightarrow{\overrightarrow{\mathbf{P}}}_{a}+\rho_{a}\left(e_{a}+k_{a}\right) \overrightarrow{\mathbf{v}}_{S}\right]_{-}^{+} \cdot \overrightarrow{\mathbf{N}}-\rho_{a}\left(e_{a}+k_{a}\right) V_{S \perp}=0} \\
\overrightarrow{\overrightarrow{\mathbf{P}}}_{a}{ }^{c h} \cdot \overrightarrow{\mathbf{N}}-\frac{V_{S \perp}}{c} \overrightarrow{\mathbf{E}}_{a} \times \overrightarrow{\mathbf{H}}_{a}=\overrightarrow{\mathbf{0}} \\
\overrightarrow{\mathbf{J}}_{E a}{ }^{c h} \cdot \overrightarrow{\mathbf{N}}-V_{S \perp} E_{a}{ }^{c h}=0
\end{array}\right. \\
& \left\{\begin{array}{l}
\overrightarrow{\overrightarrow{\mathbf{P}}}_{a} \cdot \overrightarrow{\mathbf{N}}=\mathbf{0}, \overrightarrow{\overrightarrow{\mathbf{P}}}_{a}^{c h} \cdot \overrightarrow{\mathbf{N}}=\mathbf{0} \\
\overrightarrow{\mathbf{q}}_{a} \cdot \overrightarrow{\mathbf{N}}=0, \overrightarrow{\mathbf{E}}_{a}{ }^{2}-\overrightarrow{\mathbf{B}}_{a}{ }^{2}=0
\end{array}\right.
\end{aligned}
$$




\subsection{Bilan d'impulsion-énergie à l'interface}

Nous donnons ci-après les équations de bilan, dans lesquelles les grandeurs électromagnétiques sont globalisées pour un mélange, comme l'est aussi d'ailleurs le tenseur des pressions.

○ Bilan 4D d'impulsion-énergie à l'interface :

$$
\begin{aligned}
& 4 \vec{\nabla}_{S} \cdot\left({ }_{4} \overrightarrow{\mathcal{P}}_{a}^{c h}+{ }_{4} \overrightarrow{\overrightarrow{\mathcal{P}}}_{a}^{m}\right)+\rho_{a} \overrightarrow{\mathbf{v}}_{S} \vec{\nabla}_{S} \cdot{ }_{4} \overrightarrow{\mathbf{V}}_{S}+ \\
& {\left[\overrightarrow{\overrightarrow{\mathcal{P}}}^{c h}+{ }_{4} \overrightarrow{\overrightarrow{\mathcal{P}}}^{m}+(1 / c) \rho\left(\overrightarrow{\mathbf{v}}-\overrightarrow{\mathbf{v}}_{S}\right) \otimes(\overrightarrow{\mathbf{v}}-\overrightarrow{\mathbf{V}})\right]^{+} \cdot \overrightarrow{\mathbf{N}}={ }_{4} \vec{\Phi}_{a}}
\end{aligned}
$$

Soit, en écriture classique, $k$ désignant l'espèce chimique :

$$
\begin{aligned}
& \rho_{a} \frac{d_{S} \overrightarrow{\mathbf{v}}_{S}}{d t}+\vec{\nabla}_{S} \cdot \overrightarrow{\overrightarrow{\mathbf{P}}}_{a}+\left[\overrightarrow{\overrightarrow{\mathbf{P}}}+\rho\left(\overrightarrow{\mathbf{v}}-\overrightarrow{\mathbf{v}}_{S}\right)+\overrightarrow{\overrightarrow{\mathbf{P}}}^{c h}-\frac{1}{c}(\overrightarrow{\mathbf{E}} \times \overrightarrow{\mathbf{B}}) \otimes \overrightarrow{\mathbf{V}}\right]_{-}^{+} \cdot \overrightarrow{\mathbf{N}}=\sum_{k} \rho_{a k} \vec{f}_{k a} \\
& \rho_{a} \frac{d_{S} e_{S}}{d t}+\vec{\nabla}_{S} \cdot \overrightarrow{\mathbf{q}}_{a}+\left[\overrightarrow{\mathbf{q}}+\rho\left(e-e_{S}\right)(\overrightarrow{\mathbf{v}}-\overrightarrow{\mathbf{V}})\right]_{-}^{+} \cdot \overrightarrow{\mathbf{N}}=-\overrightarrow{\overrightarrow{\mathbf{P}}}_{a}: \vec{\nabla}_{S} \otimes \overrightarrow{\mathbf{v}}_{S}+\sum_{k} \overrightarrow{\mathfrak{I}}_{D k a} \cdot \vec{f}_{k a} \\
& -\left[\left(\overrightarrow{\mathbf{v}}-\overrightarrow{\mathbf{v}}_{S}\right) \cdot \overrightarrow{\overrightarrow{\mathbf{P}}}+\frac{1}{2}\left(\overrightarrow{\mathbf{v}}-\overrightarrow{\mathbf{v}}_{S}\right)^{2}(\overrightarrow{\mathbf{v}}-\overrightarrow{\mathbf{V}})\right]_{-}^{+} \cdot \overrightarrow{\mathbf{N}}+\dot{W}_{E a}^{c h}
\end{aligned}
$$

- Espèces et masse totale :

$$
\begin{aligned}
& \frac{d_{S}\left(\rho_{a} Y_{k S}\right)}{d t}+\rho_{a} Y_{k S} \vec{\nabla}_{S} \cdot \overrightarrow{\mathbf{V}}_{S}+\vec{\nabla}_{S} \cdot \overrightarrow{\mathbf{J}}_{\mathbf{V} k a}+\left[\vec{\jmath}_{D k}+\rho Y_{k}(\overrightarrow{\mathbf{v}}-\overrightarrow{\mathbf{V}})\right]+\overrightarrow{\mathbf{N}}=\dot{W}_{k a} \\
& \frac{d_{S} \rho_{a}}{d t}+\rho_{a} \vec{\nabla}_{S} \cdot \overrightarrow{\mathbf{V}}_{S}+[\rho(\overrightarrow{\mathbf{v}}-\overrightarrow{\mathbf{V}})]+\overrightarrow{\mathbf{N}}=0
\end{aligned}
$$

\section{- Charge électrique}

$$
\begin{aligned}
& \rho_{a} \frac{d_{S} z_{S}}{d t}+\nabla \cdot \mathbf{i}_{a}+\left[\mathbf{i}+\rho\left(z-z_{S}\right)(\mathbf{v}-\mathbf{V})\right]_{-}^{+} \cdot \mathbf{N}=0 \\
& \circ \text { Entropie : }
\end{aligned}
$$

Les question du bilan d'entropie et des lois de comportement n'ont été qu'abordées en annexe A3 dans le cadre de la TPI. Voir aussi l'exemple d'application à une interface plane particulière présenté en A4.

\section{Conclusion}

Nous avons considéré les équations de bilan aux interfaces des milieux continus en présence d'un champ électromagnétique en les exprimant dans l'espace-temps de Minkowski. Dans le cas d'un mélange, des grandeurs électromagnétiques globales (non spécifiques à chaque espèce chimique) ont été adoptées. Nous nous sommes limités au cas de vitesses faibles v/c $<<1$. La présentation dans l'espace à quatre dimensions a des avantages car elle est très condensée, en particulier pour les grandeurs électromagnétiques, mais elle peut aussi permettre de traiter des cas relativistes. 


\section{Annexes}

\section{A1. Présentation de la transformation de Lorentz}

Un point $M$ de l'espace-temps est représenté par un vecteur complexe $4 \mathbf{M}$, dont matrice ligne

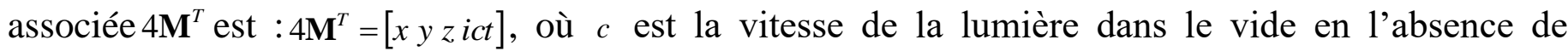
champ. Les coordonnées sont ici indiquées dans un référentiel galiléen.

Si l'on veut exprimer ces coordonnées dans un autre référentiel galiléen de vitesse relative $\overrightarrow{\mathbf{v}}$,

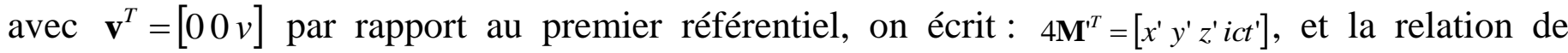
changement de référentiel se fait au moyen de la transformation spéciale de Lorentz, transformation linéaire : ${ }_{4} \mathbf{M}^{\prime}={ }_{4} \mathbf{D} \cdot{ }_{4} \mathbf{M}$, de matrice $\underline{\underline{4 \mathbf{D}}}$ :

$$
\underline{\underline{4} \mathbf{D}}=\left[\begin{array}{cccc}
1 & 0 & 0 & 0 \\
0 & 1 & 0 & 0 \\
0 & 0 & 1 / \alpha & i v / c \alpha \\
0 & 0 & -i v / c \alpha & 1 / \alpha
\end{array}\right]
$$

$\operatorname{avec}: \alpha=\sqrt{1-v^{2} / c^{2}}$.

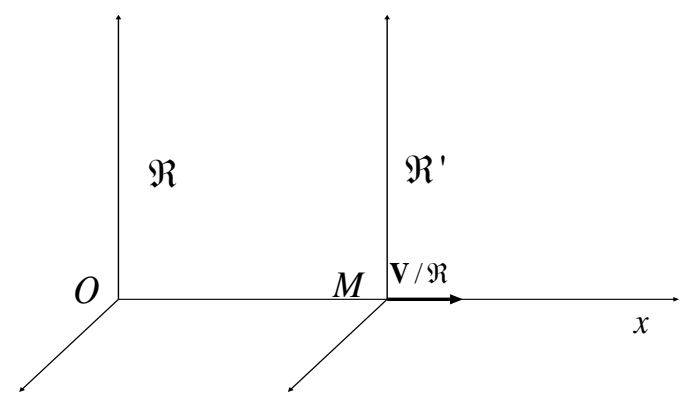

Figure A1. Référentiels galiléens. Le référentiel $\mathfrak{R}$ est celui dans lequel nous observons les événements. Au point $M$ en de vitesse instantanée $\mathbf{v}$, nous associons à l'instant $t$ le référentiel $\mathfrak{R}$ ' se déplaçant à vitesse constante $\mathbf{V}=\mathbf{v}$.

Dans l'espace à trois dimensions, la vitesse $\mathbf{v}$ d'un point matériel peut être définie à tout instant. Pour définir la vitesse comme une grandeur intrinsèque (ne dépendant pas du repère choisi pour la mesurer) dans l'espace-temps, il est nécessaire de définir un temps propre (figure A1).

A l'instant $t$, le point matériel $M$ se meut à la vitesse $\mathbf{v}(t)$ dans le référentiel $\Re$.

Considérons maintenant le référentiel Galiléen $\mathfrak{R}$ se déplaçant à vitesse constante $\mathbf{V}$ telle que, à l'instant $t$, on ait $\mathbf{v}=\mathbf{v}$. Le temps $\tau$ mesuré par rapport à un tel repère (il en existe un à tout instant) lié au point matériel est appelé temps propre de ce point matériel. Supposons que les coordonnées de $M$ restent nulles, nous aurons, dans le nouveau repère :

$$
\left[\begin{array}{l}
0 \\
0 \\
0 \\
i c \tau
\end{array}\right]=\underline{=}\left[\begin{array}{l}
0 \\
0 \\
z \\
i c t
\end{array}\right], \text { soit : }\left\{\begin{array}{l}
0=z-v t \\
c \tau=\frac{1}{\alpha}\left(-\frac{v}{c} z+c t\right)
\end{array}\right.
$$

On en déduit l'expression du temps propre du point matériel : $\tau=\frac{t}{\alpha}\left(1-\frac{v^{2}}{c^{2}}\right)=\alpha t$ 
Notons qu'ici $v$ et $\alpha$, bien que variables, doivent être considérés comme constants lorsqu'il s'agit du repère Galiléen coïncidant avec $M$ et ayant même vitesse que $M$ à l'instant $t$. La relation précédente est donc en fait une relation différentielle reliant le temps propre au temps dans le repère $\mathfrak{R}$ et doit s'écrire en toute rigueur : $d \tau=\alpha d t$.

Le quadri vecteur $4 \mathbf{V}$ est bien un 4-vecteur de l'espace de Minkowski comme on peut le vérifier aisément (réf. iv).

\section{A2. Application de la transformation de Lorentz aux grandeurs électromagnétiques}

Affectons des indices $0, \mathrm{v}$ et $\mathrm{V}$, les valeurs prises dans les référentiels au repos, suivant le mouvement particulaire et suivant un mouvement quelconque (pouvant être celui de la zone interfaciale), respectivement.

La transformation de Lorentz s'applique aux quadri-tenseurs ${ }_{4} \overrightarrow{\overrightarrow{\mathcal{F}}},{ }_{4} \overrightarrow{\overrightarrow{\mathcal{F}}} *$ et ${ }_{4} \overrightarrow{\overrightarrow{\mathcal{H}}}$ avec les formules suivantes pour les champs et les déplacements où $\mathrm{V}$ peut être remplacé par $\mathrm{v}$ :

$$
\begin{array}{ll}
\overrightarrow{\mathbf{D}}_{\mathbf{v}}=\overrightarrow{\mathbf{D}}_{\mathbf{0}}+(1 / c) \overrightarrow{\mathbf{V}} \times \overrightarrow{\mathbf{H}}_{\mathbf{0}}, & \overrightarrow{\mathbf{E}}_{\mathbf{V}}=\overrightarrow{\mathbf{E}}_{\mathbf{0}}+(1 / c) \overrightarrow{\mathbf{V}} \times \overrightarrow{\mathbf{B}}_{\mathbf{0}}, \\
\overrightarrow{\mathbf{H}}_{\mathbf{v}}=\overrightarrow{\mathbf{H}}_{\mathbf{0}}+(1 / c) \overrightarrow{\mathbf{V}} \times \overrightarrow{\mathbf{D}}_{\mathbf{0}}, & \overrightarrow{\mathbf{B}}_{\mathbf{v}}=\overrightarrow{\mathbf{B}}_{\mathbf{0}}+(1 / c) \overrightarrow{\mathbf{V}} \times \overrightarrow{\mathbf{E}}_{\mathbf{0}}
\end{array}
$$

Pour les composantes du 4-tenseur d'impulsion-énergie, la relation fondamentale entre les flux $\begin{aligned} \operatorname{devient}: & \overrightarrow{\overrightarrow{\mathbf{P}}}_{\mathbf{0}}=\overrightarrow{\overrightarrow{\mathbf{P}}}_{v}+(\overrightarrow{\mathbf{v}} / c) \otimes \overrightarrow{\mathbf{E}}_{v} \times \overrightarrow{\mathbf{H}}_{v}=\overrightarrow{\overrightarrow{\mathbf{P}}}_{\mathbf{V}}+(\overrightarrow{\mathbf{V}} / c) \otimes \overrightarrow{\mathbf{E}}_{\mathbf{V}} \times \overrightarrow{\mathbf{H}}_{\mathbf{v}} \\ & \overrightarrow{\mathbf{J}}_{\mathbf{E 0}}=\overrightarrow{\mathbf{J}}_{\mathbf{E v}}+\overrightarrow{\mathbf{v}} \mathbf{E}_{c h}=\overrightarrow{\mathbf{J}}_{\mathbf{E V}}+\overrightarrow{\mathbf{V}} \mathbf{E}_{c h}\end{aligned}$

\section{A3. Les lois de comportement à l'interface}

Le problème des lois de comportement, dans le volume et à l'interface est assez complexe, en particulier en présence de surfaces. Il faut d'une part connaître les lois d'état et d'autre part établir les lois complémentaires.

Dans les plasmas raréfiés en présence de champs électromagnétiques, les seules équations des milieux continus ne suffisent plus et il intervient des longueurs caractéristiques d'origine microscopique telles la longueur de Debye ou le rayon de Larmor. D'autre part les champs agissent différemment sur chaque espèce, il y a donc de la place pour les théories microscopiques ${ }^{\mathrm{vi}}$.

On peut néanmoins tenter d'utiliser notre théorie macroscopique et les principes de la thermodynamique irréversible (TPI) pour obtenir les lois complémentaires ${ }^{\text {vii, viii, ix }}$

Pour cela il nous faut d'abord déterminer le taux de production d'entropie, puis faire apparaitre des flux et des forces généralisées qui seront ensuite supposées vérifier des relations linéaires avec des coefficients phénoménologiques. Pour la capillarité on utilise la théorie du second gradient [réf. iv].

Sinon, on a recours à l'analyse à l'échelle moléculaire. En présence de champs électromagnétique interviennent des longueurs caractéristiques d'origine microscopique telles les longueurs de Debye et de Larmor.

L'équation locale de bilan de l'entropie a la forme suivante :

$$
\rho_{a} \frac{d s_{S}}{d t}+\vec{\nabla}_{S} \cdot \overrightarrow{\mathbf{J}}_{S a}+\left[\frac{\overrightarrow{\mathbf{q}}}{T}-\sum_{k} \overrightarrow{\mathcal{J}}_{D k} \frac{g_{k}}{T}+\rho\left(s-s_{S}\right)(\overrightarrow{\mathbf{v}}-\overrightarrow{\mathbf{V}})\right]_{-}^{+} \cdot \overrightarrow{\mathbf{N}}=\dot{W}_{S a}
$$


L'expression du taux de production d'entropie $\dot{W}_{S a}$ se déduit des autres équations de bilan et des lois d'état. Il s'écrit :

$$
\begin{aligned}
& \dot{W}_{S a}=\overrightarrow{\mathbf{q}}_{a} \cdot \vec{\nabla}_{S}\left(\frac{1}{T_{S}}\right)-\frac{1}{T_{S}} \sum_{k} \overrightarrow{\boldsymbol{\jmath}}_{D k a} \cdot\left(T_{S} \vec{\nabla}_{S}\left(\frac{g_{k S}}{T_{S}}\right)-\vec{f}_{k a}\right)-\frac{1}{T_{S}} \overrightarrow{\overrightarrow{\mathbf{\Pi}}}_{a}: \vec{\nabla}_{S} \otimes \overrightarrow{\mathbf{v}}_{S} \\
& -\sum_{k} \frac{g_{k S}}{T_{S}} \dot{W}_{k a}+\left[\left(\frac{1}{T}-\frac{1}{T_{S}}\right) \overrightarrow{\mathbf{q}}-\sum_{k}\left(\frac{g_{k}}{T}-\frac{g_{k S}}{T_{S}}\right) \overrightarrow{\boldsymbol{\jmath}}_{D k a}-\frac{1}{T}\left(\overrightarrow{\mathbf{v}}-\overrightarrow{\mathbf{v}}_{S}\right) \cdot \overrightarrow{\overrightarrow{\boldsymbol{\Pi}}}\right. \\
& \left.+\frac{\rho}{T}\left(\left(T_{S}-T\right)_{S}+\sum_{k}\left(g_{k S}-g_{k}\right) Y_{k}-\frac{1}{2}\left(\overrightarrow{\mathbf{v}}-\overrightarrow{\mathbf{v}}_{S}\right)^{2}\right)(\overrightarrow{\mathbf{v}}-\overrightarrow{\mathbf{v}})\right]_{-}^{+} \cdot \overrightarrow{\mathbf{N}}+\dot{W}_{S a}(c h)
\end{aligned}
$$

avec la partie due aux champs reliée au taux de production d'énergie interne de ces champs :

$$
\dot{W}_{E a}^{c h}=-\dot{W}_{\psi a}-\frac{\partial E a^{c h}}{\partial t}+\vec{\nabla}_{S} \cdot \overrightarrow{\mathbf{J}}_{E a}^{c h}-\left[\overrightarrow{\mathbf{J}}_{E}^{c h}\right]^{+} \cdot \overrightarrow{\mathbf{N}}+\overrightarrow{\mathbf{v}}_{S} \cdot\left(\vec{\nabla} \cdot \overrightarrow{\overrightarrow{\mathbf{P}}}_{a}^{c h}+\left[\overrightarrow{\overrightarrow{\mathbf{P}}}^{c h}\right]_{-}^{+} \cdot \overrightarrow{\mathbf{N}}\right)
$$

contenant un terme de production de potentiel $\dot{W}_{\psi a}$.

\section{A4. Exemples d'application ${ }^{x}$}

- Le travail de mise en forme du taux de production d'entropie reste à faire dans le cas général. Il a été réalisé dans des cas particuliers tels que l'effet Peltier.

- Il a également été réalisé, dans le cas de l'interface métal-plasma, en présence de réactions de surface, en utilisant des schémas réactifs et des lois de cinétique chimique pas nécessairement linéaires ${ }^{\mathrm{xi}}$.

- Les géométries des interfaces étudiées sont le plus souvent planes.

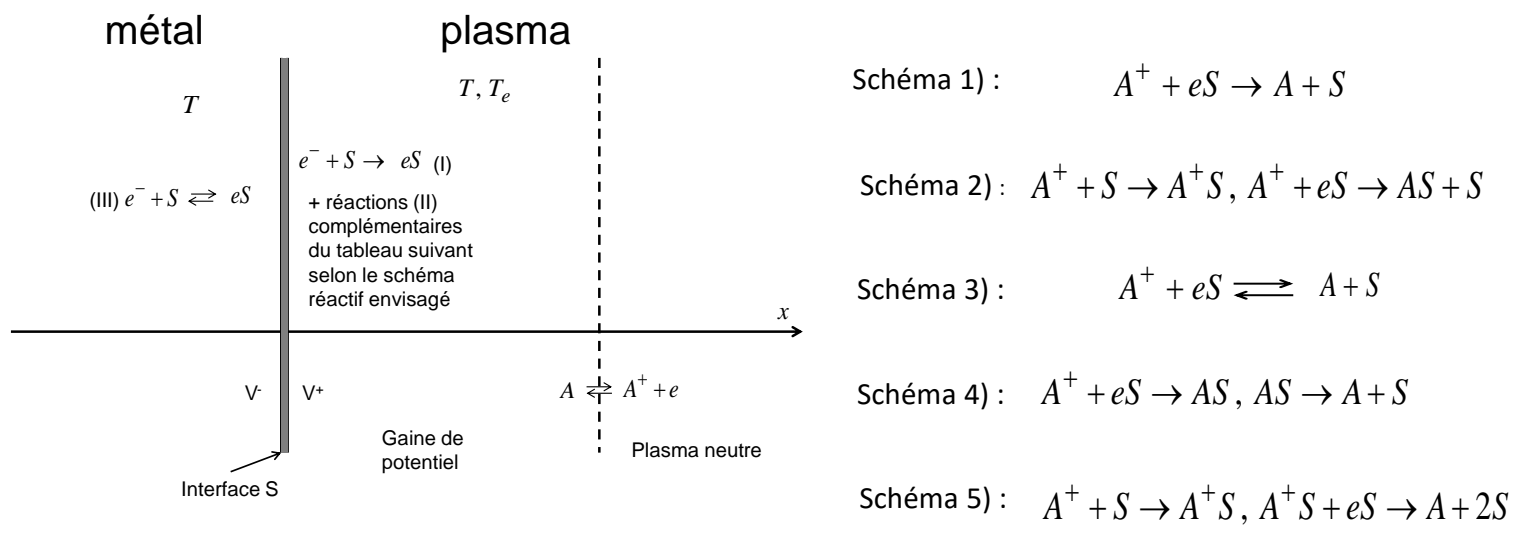

Figure A4. Modélisations de l'interface métal-plasma sous basse pression envisagée dans l'étude d'une sonde de Langmuir. 


\section{Références}

' Einstein A. Zur Elektrodynamik bewegter Körper, Annalen der Physik, vol. 17, p. 891-921, 1905.

ii Landau L., Lifchitz E., Physique théorique, vol. 2 : Théorie des champs, Éd. MIR, Moscou, 1982.

iii Prud'homme R. : Ecoulements et Réactions chimiques 3 : applications aux mélanges hétérogènes réactifs collection mécanique des fluides - Hermès-Lavoisier, Cachan, 2014. ISBN 978-2-7462-4586-0.

iv Gatignol R. \& Prud'homme R. : Mechanical and thermodynamical modeling of fluid interfaces, Series on Advances in Mathematics for Applied Sciences - Vol. 58, World Scientific, 2001. ISBN 9810243057.

"Prud'homme, R.: Flows and Chemical Reactions in an Electromagnetic Field, Fluid Mechanics series, ISTE - Wiley, 2014. ISBN 978-1-84821-78-7.

vi Barral S., Miedzik J., "Numerical investigation of closed-loop control for Hall accelerators", Journal of Applied Physics, vol. 109, p. 013302, 2011.

vii De Groot S.R., The Maxwell equations, North-Holland Publishing Company, 1969.

viii De Groot S.R. \& Mazur P., Non equilibrium thermodynamics, Dover Publications, INC., New York, 1984.

${ }^{i x}$ Kluitenberg C.A., On dielectric and magnetic relaxation phenomena and non-equilibrium thermodynamics, Physica, vol. 68, p. 75-92, 1973.

× Dudeck M., "Études thermodynamiques de phénomènes électromagnétiques interfaciaux ", Thèse de doctorat d'État, Université Pierre et Marie Curie (Paris 6), $1^{\text {er }}$ juillet 1982.

xi Dudeck M., "Evolution thermique d'une sonde électrostatique dans un gaz raréfié et partiellement ionisé», International Journal of Heat and Mass Transfer, vol. 30, №3, p. 551-557, 1987. 